\title{
Delivering hydrophilic and hydrophobic chemotherapeutics simultaneously by magnetic mesoporous silica nanoparticles to inhibit cancer cells
}

\author{
This article was published in the following Dove Press journal: \\ International Journal of Nanomedicine \\ 22 February 2012 \\ Number of times this article has been viewed
}

\author{
Qian Liu* \\ Jixi Zhang* \\ Wei Sun \\ Qian Reuben Xie \\ Weiliang Xia \\ Hongchen $\mathrm{Gu}$
}

School of Biomedical Engineering and Med-X Research Institute,

Shanghai Jiao Tong University,

Shanghai, People's Republic

of China

*These authors contributed equally to this work
Correspondence: Hongchen Gu School of Biomedical Engineering and Med-X Research Institute, Shanghai Jiao Tong University, 1954 Huashan Road, Shanghai, 200030, People's Republic of China

Tel +862162933176

Fax +86 2I 62932907

Email hcgu@sjtu.edu.cn

Weiliang Xia

School of Biomedical Engineering and Med-X Research Institute, Shanghai Jiao Tong University, 1954 Huashan Road, Shanghai, 200030, People's Republic of China

$\mathrm{Tel}+862$ I 6293329 |

Fax +86 21 92932302

Email wlxia@sjtu.edu.cn

\begin{abstract}
Using nanoparticles to deliver chemotherapeutics offers new opportunities for cancer therapy, but challenges still remain when they are used for the delivery of multiple drugs, especially for the synchronous delivery of hydrophilic and hydrophobic drugs in combination therapies. In this paper, we developed an approach to deliver hydrophilic-hydrophobic anticancer drug pairs by employing magnetic mesoporous silica nanoparticles (MMSNs). We prepared $50 \mathrm{~nm}$-sized MMSNs with uniform pore size and evaluated their capability for the loading of two combinations of chemotherapeutics, namely doxorubicin-paclitaxel and doxorubicinrapamycin, by means of sequential adsorption from the aqueous solution of doxorubicin and nonaqueous solutions of paclitaxel or rapamycin. Experimental results showed that the present strategy successfully realized the co-loading of hydrophilic and hydrophobic drugs with high-loading content and widely tunable ratio range. We elaborate on the theory behind the molecular interaction between the silica hydroxyl groups and drug molecules, which underlie the controllable loading, and the subsequent release of the drug pairs. Then we demonstrate that the multidrug-loaded MMSNs could be easily internalized by A549 human pulmonary adenocarcinoma cells, and produce enhanced tumor cell apoptosis and growth inhibition as compared to single-drug loaded MMSNs. Our study thus realized simultaneous and dose-tunable delivery of hydrophilic and hydrophobic drugs, which were endowed with improved anticancer efficacy. This strategy could be readily extended to other chemotherapeutic combinations and might have clinically translatable significance.
\end{abstract}

Keywords: co-delivery, combination chemotherapy, doxorubicin, mesoporous nanoparticles

\section{Introduction}

The integration of drug delivery with nanotechnology in the field of cancer therapy has attracted much attention in recent decades. ${ }^{1-5}$ By improving drug bioaccessibility and chemosensitivity, nanoparticles as drug-delivery systems (DDSs) could increase therapeutic efficacy and mitigate side effects. ${ }^{6,7}$ Among the recent breakthroughs that brought new exciting possibilities to this area, mesoporous silica nanoparticles (MSNs) have been highlighted and shown to be a promising candidate for a cancer cell-specific delivery vehicle..$^{8-11}$ These silica-based nanoparticles possess a large number of mesoporous channels which endow them with unique structural features including uniform and tunable pore size $(2-15 \mathrm{~nm})$, high surface area (over $\left.700 \mathrm{~m}^{2} / \mathrm{g}\right)$, large pore volume (ranges from $0.6-1 \mathrm{~cm}^{3} / \mathrm{g}$ ) and readily functionalized surface. ${ }^{12}$ In vitro and in vivo studies demonstrated that MSNs possessed excellent biocompatibility, and could be 
degraded and metabolized in a relatively short term (over $90 \%$ were degraded within 96 hours). ${ }^{13,14}$ These features make it possible to reach high drug-loading capacity and allow for applying a smaller dose of nanoparticles to minimize particleinduced toxicity. Furthermore, the controlled release of the loaded therapeutic cargoes from MSNs can be easily achieved by either a diffusion-dependent process or a stimulus responsive device at the pore openings. ${ }^{15,16}$ Additionally, the mesopores could also protect bioactive drugs from undesired enzymatic degradation before reaching the target, owing to the inaccessibility of the inner surface to the enzyme in blood or tissue plasma. ${ }^{17}$ Consequently, a variety of cancer therapeutic agents have been successfully delivered by MSNs, such as chemotherapeutics, ${ }^{18,19}$ small interfering RNAs (siRNAs) ${ }^{20,21}$ and proteins. ${ }^{22}$

Since cancer is a complex disease that arises with the involvement of multiple factors in multiple steps, the design of a treatment will need to address these concerns, such as targeting the so-called hallmark features of cancers. ${ }^{23}$ By targeting the key node or pathway essential for cancer cell survival or resistance to treatment, we might be able to suppress or eradicate cancers more efficiently. In this regard, delivering multiple drugs instead of a single therapeutic agent is supposed to achieve more significant cancer inhibition. ${ }^{24-26}$ For example, Olive and colleagues combined gemcitabine with IPI-926 (a Hedgehog signaling pathway inhibitor that depletes tumor-associated stromal tissue) produced an increased anticancer activity in a pancreatic ductal adenocarcinoma mouse model. ${ }^{27}$ In a clinical trial, patients with metastatic renal cell carcinoma had benefited from the combination of bevacizumab (a vascular endothelial growth factor inhibitor) and everolimus (a mammalian target of rapamycin [mTOR] inhibitor). ${ }^{28}$ In addition, other strategies that combine chemotherapeutics with the inhibition of the multidrug resistance (MDR) or antiapoptosis genes can also yield improved efficacy, as illustrated by recent studies where MSN-based DDSs were used to deliver siRNAs that specifically suppressed the MDR pump activity or apoptosis resistance and an anticancer drug simultaneously into cancer cells. ${ }^{29,30}$

Based on the aforementioned advantages, the use of MSNs as multidrug DDSs was regarded to improve the therapeutic index and reduce side effects. ${ }^{31,32}$ Despite this, the employment of MSNs for combination cancer therapy still meets some critical challenges. Firstly, the properties of different drugs (eg, solubility, molecular weight) vary greatly, which would handicap the drug-loading process. Secondly, it is hard to control the amount of different drugs to be loaded on nanoparticles to achieve a desirable ratio, which is important for improving the clinical effect. Notably, He and colleagues have recently demonstrated that drugs/surfactant micelles-co-loaded MSNs (drugs-micelles-MMSNs) could serve as a new type of multidrug delivery system where surfactant micelles (CTAB) was used as chemosensitizer and $\mathrm{pH}$-responsive device for the drug doxorubicin (DOX). ${ }^{33}$ Their approach offers a new strategy to co-load hydrophobic therapeutics in MSNs that induced cancer cell apoptosis synergistically. Due to this unique in-situ preparation technology, however, the surfactant CTAB could not be substituted by chemotherapeutics and the loaded cargo is limited to one anticancer drug, thus limiting the application extension of multidrug co-delivery. To the best of our knowledge, very few studies have been reported about the co-delivery of drugs with different molecular properties by surfactant-free MSNs. The main reason could be attributed to the difficulty in realization and control of co-loading in the mesoporous channels. Polymer nanoparticles and liposomes have also been used for delivery of hydrophobic and hydrophilic anticancer drugs; ${ }^{34}$ however, chemical conjugation was generally involved in the loading processes, therefore increasing the operational complexity. Meanwhile, the stoichiometry issue is another obstacle to control the dose ratio.

In this work, we developed another strategy of co-loading water soluble and poorly-soluble chemotherapeutics in mesoporous nanoparticles, which was based on our recent established magnetic MSN (MMSN) platform. ${ }^{35,36}$ We herein chose DOX, paclitaxel (PTX), and rapamycin (RAPA) as model drugs, all of which are currently anticancer drugs used in clinical practice and have typical characters of solubility. By taking advantages of the mesoporous properties of MMSNs and a sequential adsorption of the drugs from their aqueous and nonaqueous solutions, the DOX-PTX and DOX-RAPA combinations shows high loading capacities on MMSNs, as well as an interactive adsorption behavior where the adsorption of DOX is affected by that of PTX/RAPA (Figure 1). The possibility of tuning the dose ratio of loading drugs to a desirable value had been explored. Furthermore, the cellular uptake of the drug-loaded MMSNs prepared by this approach, namely, DOX-RAPA-MMSNs and DOX-PTX-MMSNs, and the resulting cancer cell suppression had also been evaluated.

\section{Material and methods \\ Materials}

Cetyltrimethylammonium bromide (CTAB), 3-aminopropyltri-ethoxysilane (APTES), tetraethylorthosilicate (TEOS), and ammonium nitrate $\left(\mathrm{NH}_{4} \mathrm{NO}_{3}\right)$, these used reagents were 


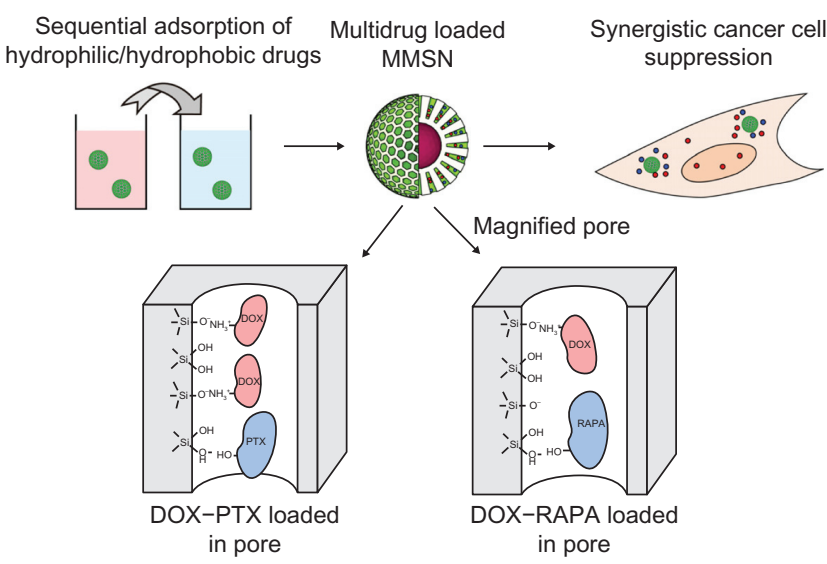

Figure I The representative scheme of loading multidrugs in MMSNs. The multidrugloaded MMSNs were prepared by a sequential adsorption procedure, and induced synergistic cancer cell suppression. DOX adsorb on MMSNs via electrostatic attraction, while PTX and RAPA through hydrogen bond or polar interactions. The adsorption of drugs was affected by each other.

Abbreviations: DOX, doxorubicin; MMSNs, magnetic mesoporous silica nanoparticles; PTX, paclitaxel; RAPA, rapamycin

analytical reagent grade; fluorescein isothiocyanate isomer I (FITC $\geq 98 \%$ ). Deionized water was used.

\section{Synthesis of MMSNs}

MMSNs were synthesized based on our procedure published previously. ${ }^{36}$ Briefly, magnetic nanoparticles were synthesized by a coprecipitation approach with a ratio of $0.6 \mathrm{mg}$ in $1 \mathrm{~mL}$ chloroform, stabilized by oleic acid, and then dispersed in $0.74 \mathrm{~mL}$ chloroform, followed by addition of $5 \mathrm{~mL}$ aqueous solution containing $0.15 \mathrm{~g}$ CTAB. After vigorously stirring, homogeneous oil-in-water microemulsion was obtained, which was then stirred at $70^{\circ} \mathrm{C}$ for 10 minutes to remove the remaining chloroform via evaporation. ${ }^{37}$ The water-dispersed nanoparticles were obtained. Meanwhile, FITC and APTES were reacted in $0.5 \mathrm{~mL}$ ethanol under dark conditions for 2 hours to form FITC-APTES, at molar ratio of FITC:APTES $=1: 10$. The resulted nanoparticles in aqueous solution was diluted within $45 \mathrm{~mL}$ deionized water, succeeded by adding $0.3 \mathrm{~mL} \mathrm{NaOH}$ solution $(2 \mathrm{M}), 0.5 \mathrm{~mL}$ TEOS, $0.5 \mathrm{~mL}$ of FITC-APTES solution, and $3 \mathrm{~mL}$ ethyl acetate sequentially. The mixture was stirred at $600 \mathrm{rpm}$ for 3 hours at $70^{\circ} \mathrm{C}$. The resultant was collected by centrifugation and washed with water and ethanol five times. Finally, CTAB was removed by refluxing in an ethanol solution of ammonium nitrate $\left(\mathrm{NH}_{4} \mathrm{NO}_{3}, 10 \mathrm{mg} / \mathrm{mL}\right)$ for 1 hour, and repeated three times to yield FITC-labeled MMSNs.

\section{Characterization of MMSNs}

Transmission electron microscope (TEM) images were obtained by a JEM 2010 instrument (JEOL, Tokyo, Japan) with $200 \mathrm{KV}$ acceleration voltage in order to investigate the size, morphology, and integrity of the nanoparticles. The hydrodynamic size of the samples was measured using dynamic light-scattering (DLS) techniques by a Zetasizer Nano instrument (Malvern Instruments, Malvern, UK) at $298 \mathrm{~K}$. Nitrogen sorption isotherms were measured at $77 \mathrm{~K}$ with a Micromeritics ASAP 2010 analyzer (Micromeritics, Norcross, GA). The specific surface area was calculated by the Brunauer-Emmett-Teller (BET) method $^{38}$ in a linear relative pressure $(\mathrm{P} / \mathrm{P} 0)$ range between 0.05 and 0.25 . The pore size distributions were derived from the desorption branches of the isotherms by the nonlocal density functional theory method ${ }^{39}$ using the Quantachrome Autosorb software (version 1.0; (Quantachrome Instruments, Boynton Beach, FL). The total pore volume $(\mathrm{Vp})$ of the samples was derived by a single point measurement of adsorbed nitrogen amount at a relative pressure (P/P0) of 0.8 in order to exclude data from the range of interparticle spaces. Fluorescence spectrum of sample in water was detected using a LS 55 fluorescence spectrometer (Perkin Elmer, Waltham, MA) with a xenon lamp.

\section{Drug loading}

For loading of DOX, $10 \mathrm{mg}$ of MMSNs was dispersed in $2 \mathrm{~mL}$ of water/methanol (60:40, v:v) mixed solution of DOX, and the mixture was shaken at $25^{\circ} \mathrm{C}$ for 24 hours. The dispersion was centrifuged at $10,000 \mathrm{~g}$ to collect the DOX-loaded MMSNs (DOX-MMSNs), and subsequently the drug-loaded MMSNs were washed by distilled water to remove the DOX adsorbed on the exterior surface. The remaining solvent was removed from MMSNs by vacuum drying at $25^{\circ} \mathrm{C}$ for at least 8 hours. The previous adsorption supernatant and wash eluent were combined to determine the amount of DOX loaded into MMSNs. For PTX- and RAPA-loading, empty and DOXloaded MMSNs were suspended in $10 \mathrm{~mL}$ PTX or RAPA solution in tetrachloromethane, and the mixtures were shaken at $25^{\circ} \mathrm{C}$ for 24 hours. After that, the drug-loaded MMSNs were separated by centrifugation and the remaining solvent was removed by vacuum drying. Thereafter, drug-loaded MMSNs, ie, DOX-MMSNs, PTX-MMSNs, DOX-PTXMMSNs and DOX-RAPA-MMSNs were prepared.

\section{Drug-releasing studies}

Drug-loaded nanoparticles were dispersed in $2 \mathrm{~mL}$ of phosphate-buffered saline (PBS), and the dispersion was loaded in a dialysis bag with a molecular weight cutoff of 8000 Dalton. The dialysis bag was then kept in $8 \mathrm{~mL}$ PBS and gently shaken at $37^{\circ} \mathrm{C}$. At different time intervals, $1 \mathrm{~mL}$ of the dialysate was taken out to test the amount of 
released drugs. Fresh PBS was added to the dialysate to remain the constant volume.

\section{Determination of drug concentration}

DOX and RAPA concentrations were determined via analyzing the UV-Vis at $480 \mathrm{~nm}$ and $280 \mathrm{~nm}$ on a NanoDrop 1000 spectrophotometer (Thermo Scientific, Rochester, NY), respectively.

The PTX concentration was measured on an Agilent 1100 Series high-pressure liquid chromotograph (Agilent Technologies, Santa Clara, CA) equipped with a C18 reverse phase column (Cosmosil C18 AR-II; Nacalai, Tokyo, Japan). The analysis was taken at flowing rate of $1 \mathrm{~mL} / \mathrm{min}$ by using $65 \%$ of methanol/water solution (v/v) as mobile phase, and PTX was determined at $227 \mathrm{~nm}$ of UV-Vis adsorption wavelength.

\section{Cell culture}

Human pulmonary adenocarcinoma cells (A549) were cultured in Dulbecco's modified Eagle's medium (DMEM), supplemented with $10 \%$ fetal bovine serum, $100 \mu \mathrm{g} / \mathrm{mL}$ penicillin, and streptomycin in $37^{\circ} \mathrm{C}$ humidity atmosphere contains $5 \% \mathrm{CO}_{2}$. The culture medium was changed every 2 days before experimental operation.

\section{Cellular uptake of MMSNs}

Cells were seeded on cover slips placed in a six-well plate, and incubated in $37^{\circ} \mathrm{C}$ incubator. Twenty-four hours later, cells were treated by MMSNs. After culture in $37^{\circ} \mathrm{C}$ humidity incubator, cells were washed with PBS thoroughly, fixed by $4 \%$ paraformaldehyde for 15 minutes, and then treated by 4',6-diamidino-2-phenylindole (DAPI) solution for nuclear staining. These cover slips were sealed and performed on a Leica confocal microscopy system (TCS SP5; Leica, Mannheim, Germany).

\section{Apoptosis assay}

Cell apoptosis was determined by fluorescence-activated cell sorting (FACS) with an Annexin V apoptosis detection kit (SouthernBiotech, Birmingham, AL). A549 cells were seeded on six-well plate at $1 \times 10^{6}$ per well, and exposed to DMEM medium containing $50 \mu \mathrm{g} / \mathrm{mL}$ empty or drug-loaded MMSNs for 24 hours. Cells were then washed by PBS and harvested by trypsinization. After being labeled by Annexin V-PE and 7AAD according to the manufacturer's protocol, cells were immediately analyzed using a flow cytometer (FACSAria II; BD Biosciences, San Jose, CA).

\section{Cell proliferation and survival}

A549 cells were cultured in a 96-well plate with 5,000 cells/ well. Twenty-four hours later, the culture medium was changed with drug-loaded MMSNs. After 48 hours of exposure, a cellcounting kit-8 (CCK-8) (Sigma Aldrich, St Louis, MO) was used to measure the cell viability according to the manufacturer's protocol. The optical absorption of each well at $450 \mathrm{~nm}$ was read by a microplate reader (BioTek, Seattle, WA).

\section{Statistical analysis}

Each experiment was carried out in triplicate at least. Data are presented as means \pm standard deviation. Differences between mean values of two groups and more than two groups were tested for significance by Student's $t$-test. $P \leq 0.05$ was considered significant.

\section{Results and discussion}

\section{Synthesis and characterization of MMSNs}

MMSNs were synthesized by the base-catalyzed and liquid phase-seeded growth approach using a CTAB-terminated $\mathrm{Fe}_{3} \mathrm{O}_{4}$ nanocrystal as the seed to edify the mesoporous shell. As shown in the typical TEM image of the MMSNs (Figure 2A), the obtained material is composed of monodispersed nanospheres $\sim 50 \mathrm{~nm}$ in size. The MMSN contains a single $\mathrm{Fe}_{3} \mathrm{O}_{4}$ nanocrystal core wrapped evenly by the mesoporous shell and the radially aligned mesopores are clearly observed in the shell. The observed pore structure is supported by nitrogen adsorption/desorption isotherm as shown in Figure 2B. The isotherm is of type IV according to the International Union of Pure and Applied Chemistry classification, which is typical for a mesoporous material. There are two capillary condensation steps at $\mathrm{P} / \mathrm{P} 0 \approx 0.3-0.4$ and $\mathrm{P} / \mathrm{P} 0 \approx 0.9$, which correspond to the mesopores and textual porosity produced by interparticle packing. The primary pore size determined from the pore size distribution curve (Figure 2B, inset) is $3.8 \pm 1.2 \mathrm{~nm}$, and the surface area and the pore volume of MMSNs are determined to be $700 \mathrm{~m}^{2} / \mathrm{g}$ and $0.44 \mathrm{~cm}^{3} / \mathrm{g}$, respectively, thus making it possible for efficient loading of sufficient amount of anticancer drugs. In addition, the hydrodynamic diameter distribution of MMSNs exhibits a value of $80 \pm 30 \mathrm{~nm}$ (Figure 2C). Due to such a small particle size, it is possible for MMSNs to leak from vasculature into tumor via the so-called enhanced permeability and retention effect, ${ }^{40}$ thus facilitating in vivo applications. The photoluminescence excitation and emission spectra demonstrated the typical feature of FITC (Figure 2D), indicative of successful incorporation of FITC in the mesoporous silica matrix. To sum up, the present synthesis technology 
A

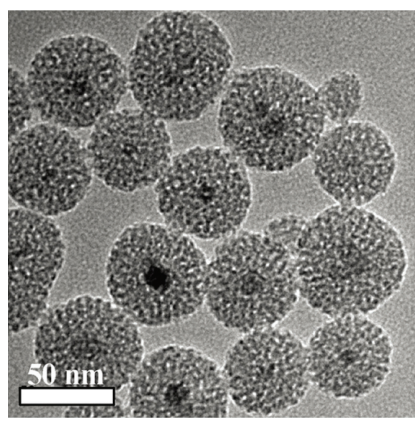

C

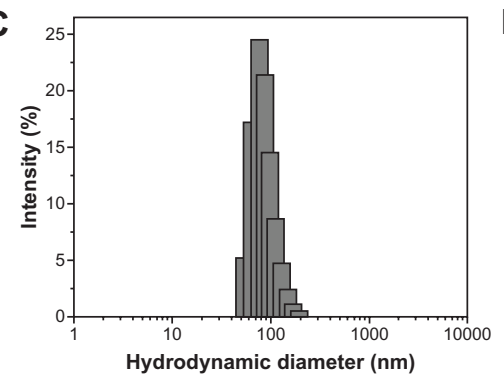

B

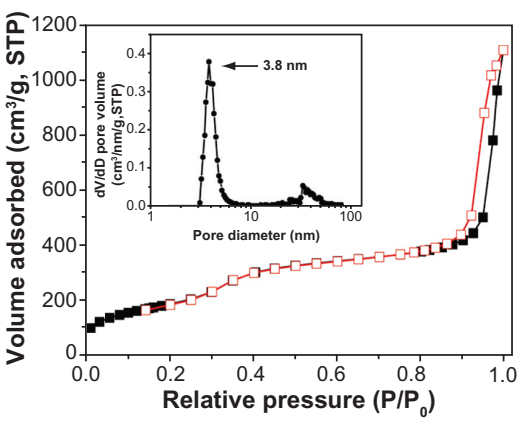

D

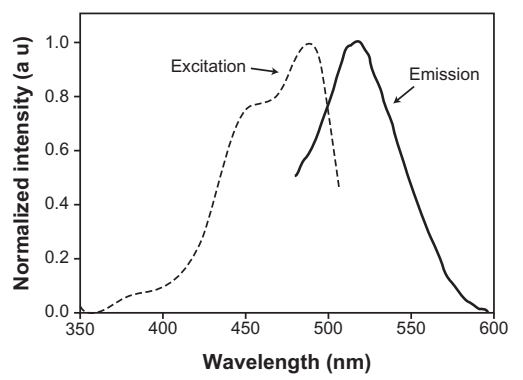

Figure 2 Characterization of the surfactant-free MMSN sample. (A) TEM image of MMSNs. (B) Nitrogen adsorption/desorption isotherm and the corresponding pore size distribution (inset). (C) Hydrodynamic diameter distribution of MMSNs. (D) Fluorescent excitation and emission spectra of FITC-MMSNs that are dispersed in PBS.

Abbreviations: FITC, fluorescein isothiocyanate; MMSNs, magnetic mesoporous silica nanoparticles; PBS, phosphate-buffered saline; STP, standard temperature and pressure; TEM, transmission electron microscope.

generated fluorescent dye-labeled MMSNs. The magnetic core imparts the MMSNs with magnetic property for magnetic resonance imaging and/or magnetic field manipulation in the further biological applications, whereas the fluorescent dye makes it convenient to track the particles and investigate cellular uptake.

\section{Chemotherapeutics co-loading strategy}

We chose three model drugs to explore the ability of MMSNs for synchronously loading hydrophobic and hydrophilic chemotherapeutic. DOX was chosen as the hydrophilic model drug, as it is broadly used for first-line chemotherapy. Besides, DOX emit fluorescence when excited at $480 \mathrm{~nm}$ wavelength, which could facilitate our following study. PTX, as a hydrophobic drug, is a routine anticancer agent broadly used in clinical treatment. RAPA, another hydrophobic drug, was also chosen as a model drug in our study. RAPA was initially used as an immunosuppressive agent, but was recently identified to possess an antiproliferative effect, and its cancer inhibition efficiency was revealed in clinical trials. ${ }^{41}$ DOX was combined with PTX or RAPA to form hydrophilichydrophobic pairs.

DOX has a large solubility in aqueous conditions while being almost dissoluble in dichloromethane or tetrachloromethane. In contrast, PTX and RAPA have poor solubility in aqueous liquids. Therefore, the different dissolving rates led us to employ a sequential-loading approach.
Adsorption isotherms for DOX, RAPA, and PTX to MMSNs, determined at $298 \mathrm{~K}$ individually, are shown in Figure 3. All the individual isotherms in Figure 3A imply a typical monolayer adsorption behavior: followed by the initial increase, the adsorbed amount of drugs reached a plateau at higher equilibrium concentrations. The drastic increase of adsorbed drug amount at low concentrations indicates a high affinity characteristic for the adsorption in all cases. The maximum adsorbed amounts from the plateau values are $242.6 \pm 4.4$, $185.7 \pm 1.6$, and $156.0 \pm 3.3 \mu \mathrm{g} / \mathrm{mg}$, or surface excess values (defined as the amount of drug adsorbed per unit specific surface area of MMSNs) of $0.65,0.30$, and $0.26 \mu \mathrm{mol} / \mathrm{m}^{2}$ for DOX, RAPA, and PTX, respectively. As expected, the loading capacities of MMSNs are much higher than other nanoparticlebased delivery systems resulting from their high surface area and large pore volume. ${ }^{12}$

The co-loading of both the hydrophilic drug (DOX) and the hydrophobic drug (RAPA or PTX) in MMSNs was implemented via a sequential adsorption procedure, namely, loading DOX in aqueous solution first, followed by adsorbing RAPA (or PTX) from the nonaqueous medium. Owing to the large polarity discrepancy between the organic solvent (tetrachloromethane) and DOX, no leakage of DOX was detected in the loading process of RAPA or PTX, which makes the co-loading possible and efficient. To leave the active binding sites available for the adsorption of the hydrophobic drugs, the loading degree of DOX was controlled by varying the 

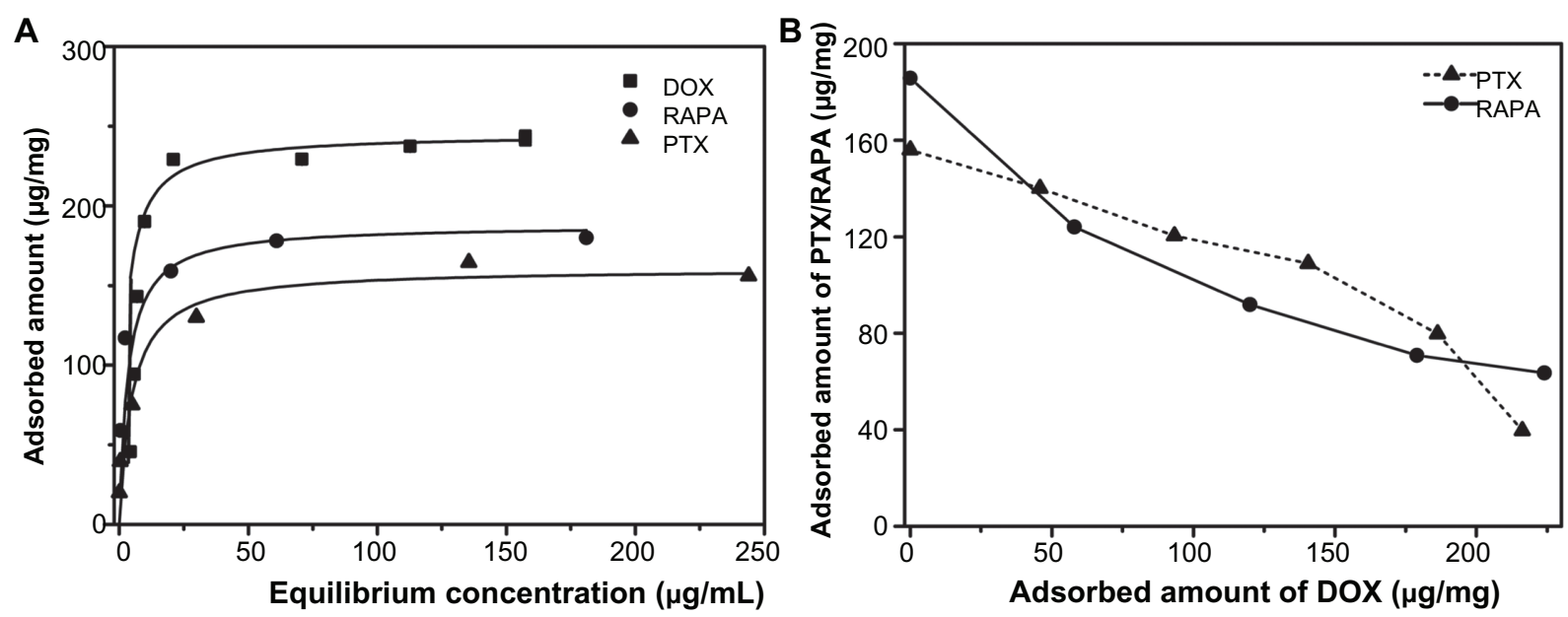

C

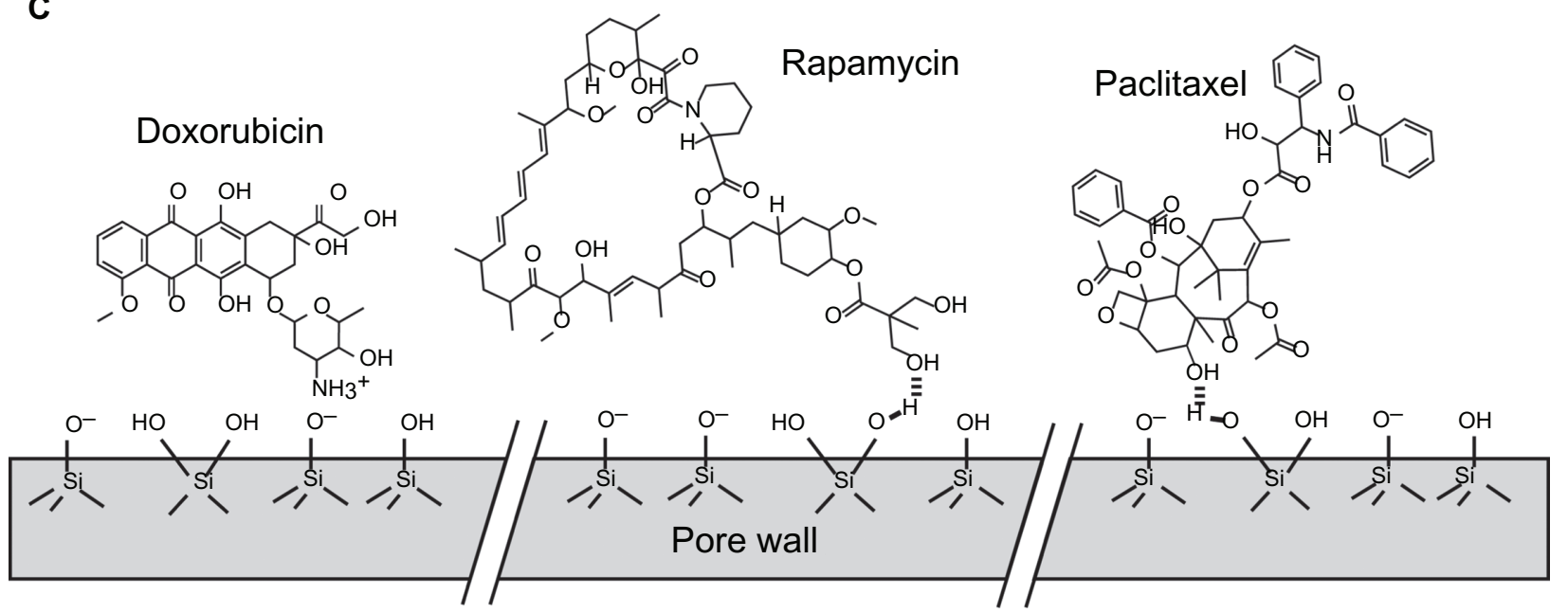

Figure 3 Adsorption profiles of DOX, RAPA, and PTX on MMSNs. (A) Adsorption isotherms for DOX, RAPA, and PTX determined at 298 K. (B) The loading-dependence curve between the hydrophobic drug (RAPA or PTX) and DOX. (C) The adsorption interactions between DOX, PTX, RAPA, and mesoporous silica matrix. DOX were attracted to the silica matrix through electrostatic interaction, while PTX and RAPA were attracted through hydrogen bonding and polar interactions.

Abbreviations: DOX, doxorubicin; MMSNs, magnetic mesoporous silica nanoparticles; PTX, paclitaxel; RAPA, rapamycin.

initial concentrations below the saturation adsorption condition. The loading-degree dependence between the hydrophobic drug and DOX is shown in Figure 3B (see also Supplementary material, Table S1 and S2). As shown, with the increase of the preloaded DOX amount from 0 to $245 \mu \mathrm{g} /$ $\mathrm{mg}$, the RAPA-loading degree decreases from its saturation adsorption value of $185 \mu \mathrm{g} / \mathrm{mg}$ to $64 \mu \mathrm{g} / \mathrm{mg}$, while the PTXloading degree decreases from $156 \mu \mathrm{g} / \mathrm{mg}$ to $40 \mu \mathrm{g} / \mathrm{mg}$. In other words, when the loading of hydrophobic drugs become saturated following the initial DOX loading, the ratio of loaded DOX vs RAPA ranges from 0:185 to 245:64 (m:m), whereas the ratio of loaded DOX vs PTX ranges from 0:156 to $245: 40(\mathrm{~m}: \mathrm{m})$, with the overall quantity of drugs no less than $\sim 200 \mu \mathrm{g} / \mathrm{mg}$ across the full spectrum of combinations. However, the loading of hydrophobic drugs can be reduced when their adsorption is not saturated (the area below the curves). Taken together, by controlling the starting drug concentration in the two solvents, it is possible to achieve any desirable ratios of hydrophilic to hydrophobic drugs.

Regarding the above results, a conclusion can be drawn that the present strategy successfully realized the co-loading of hydrophilic and hydrophobic drug with high loading amounts and widely tunable ratio range. The co-loading strategy can thus pave a new way for the application of MMSNs in multidrug co-loading; meanwhile, it can also be generalized to the loading of other chemotherapeutic combinations composed by drugs with different molecular properties (ie, water solubility).

The above co-loading capability can be rationalized from the distinct adsorption interactions between drugs and different binding sites in mesoporous silica under the selected solvent conditions (Figure 3C); ${ }^{11}$ The drug molecules have limited number of binding sites for MMSNs (Supplementary material, Figure S1); while the accessible adsorption sites 
on both the inner and the outer pore surface of MCM-41 type mesoporous silica materials are composed of $\mathrm{Q}_{2}$ silanols $\left([\mathrm{SiO}]_{2} \mathrm{Si}[\mathrm{OH}]_{2}\right.$, with a $\mathrm{pKa}$ value around 8.5$)$ and $\mathrm{Q}_{3}$ silanols $\left([\mathrm{SiO}]_{3} \mathrm{SiOH}, \mathrm{pKa}=2\right.$ ) with different concentration proportions. ${ }^{42,43}$ The hydrophilic drug DOX has a pKa of 8.3 and thus is positively charged in the aqueous medium, while MMSNs are negatively charged (the zeta potential is $-31 \mathrm{mV}$ at $\mathrm{pH}=7$ ), thus leading to a strong electrostatic attraction to the negatively charged silanols (deprotoned $\mathrm{Q}_{3}$ silanols). For the loading of the hydrophobic drugs in nonaqueous medium, electrostatic interaction is negligible; however, hydrogen bonds or polar interactions are sufficient to provide considerable attraction of the hydrophobic drugs with polar groups to the active sites on the silica surface (both $\mathrm{Q}_{2}$ and $\mathrm{Q}_{3}$ silanols). For this reason, postloading of a hydrophobic drug can still take place in the presence of preloaded DOX. Moreover, it is noteworthy to mention again that the loading degrees of the hydrophobic drugs all declined with the increase of preloaded DOX. When the hydrophobic drugs diffused into the mesopore channels, they encounter steric hindrance from the already adsorbed DOX on the surface. At low DOX coverage, the hydrophobic molecules can more or less easily diffuse and transport in the pore channels to find available space (not already occupied by the adsorbed DOX molecules). As the drug coverage increases, the free surface reduces and it becomes increasingly difficult for these molecules to diffuse through the pore channels and adsorb onto accessible binding sites.

\section{Characterization of DOX release from MMSNs in vitro}

We next explored the release profiles of DOX-loaded MMSNs. Regarding the release properties of PTX and RAPA, because of their poor solubility in aqueous solution, the released amounts were minimal during the processing period of the in vitro experiments (less than 24 hours). ${ }^{18,44}$ So we then only investigated the in vitro release behavior of DOX in the co-loading system with the same loading degree of DOX $(\sim 100 \mu \mathrm{g} / \mathrm{mg})$, which might help us to understand the intracellular performance for the dual-drug coloaded MMSNs. The cumulative DOX release curves of DOXMMSNs, DOX-RAPA-MMSNs, and DOX-PTX-MMSNs systems in $\mathrm{PBS}$ at $37^{\circ} \mathrm{C}$ are shown in Figure 4. Initially, for approximately the first 6 hours, a rapid release of DOX could be observed in all systems. Subsequently, a stage of slow release followed up to the cumulative release time of 48 hours. For DOX-MMSNs, the DOX release amount reached $26 \%$ at 10 hours, whereas $23 \%$ and $19 \%$ of the DOX

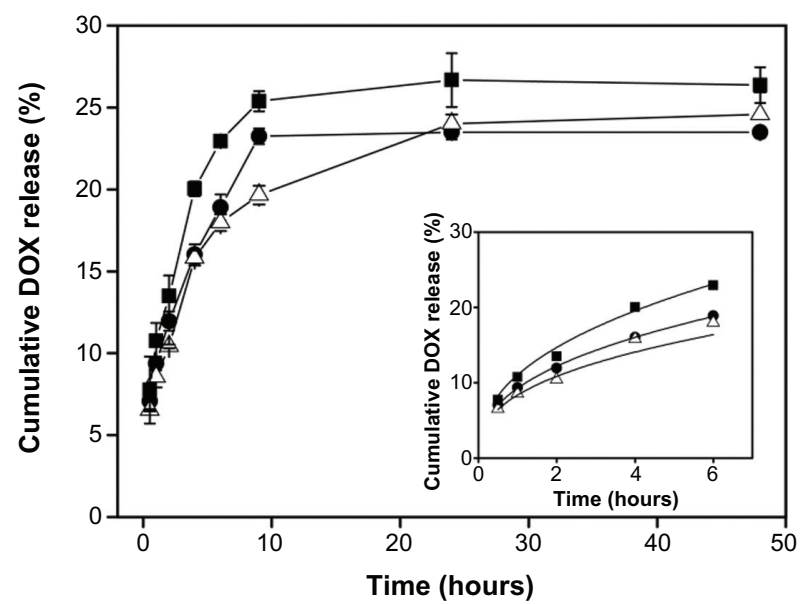

Figure 4 The release profiles of DOX on different loading fashions. DOX from individual ( $\bullet$ ), DOX-PTX co-loading $(\bullet)$, and DOX-RAPA co-loading $(\Delta)$. The releasing experiment was carried out in PBS at $310 \mathrm{~K}$. The cumulative release of DOX is about $25 \%$ in the three different situations, but the release of DOX from the individual loading system is faster than that from the co-loaded fashion. Abbreviations: DOX, doxorubicin; PTX, paclitaxel; RAPA, rapamycin.

was released at the same time point in the case of DOX-PTXMMSNs and DOX-RAPA-MMSNs. Moreover, it took 24 hours for the amount of DOX release from DOX-RAPAMMSNs to reach as high as $24 \%$. This means that dualdelivery systems of DOX-RAPA-MMSNs and DOX-PTX-MMSNs displayed a slower release rate than that of DOX-MMSNs.

The Korsmeyer-Peppas model (Eq. 1) is a comprehensive equation to describe and explain the kinetics of drug release from the matrices. ${ }^{45,46}$ This diffusion model is expected to be valid up to approximately $60 \%$ of cumulative drug released; therefore, the data for analysis were restricted to that range.

$$
\frac{M_{t}}{M_{\infty}}=k t^{n}
$$

where $M_{t}$ and $M_{\infty}$ denote the cumulative mass of drug released at time $t$ and at infinite time, respectively; $k$ is a kinetic constant characteristic of the drug-carrier system; and $n$ is an exponent that characterizes the mechanism of drug release.

Our results indicated that the data points up to $60 \%$ of the drug release in the cumulative release curve of the coloading system were fitted in the Korsmeyer-Peppas model with good linearity and the exponent $n$ in every case was lower than 0.45. According to the Korsmeyer-Peppas model, the drug release is a simple diffusion-controlled Fickian process for our co-delivery system, which involves two processes: first, the solvent diffuses into the pores of the mesoporous silica to dissolve the drug and second, the dissolved drug molecules diffuse out of the pore. ${ }^{47}$ It has been 
reported previously that a large loading amount of hydrophobic drug ${ }^{48}$ or modifying the pore opening with a hydrophobic group after drug loading ${ }^{49}$ makes the pore surface or pore opening more hydrophobic, which could restrict the kinetics of water diffusion into the matrix and slow down the release process subsequently. In our co-delivery system, the sustained release of DOX can thus be well explained in the case of the samples with postloaded RAPA or PTX.

\section{Uptake of MMSNs and release of loaded drugs in cancer cells}

With the establishment of multidrug-loading and release profile for MMSNs, we then tested whether these particles could be readily internalized by cancer cells followed by release of the cargoes. For this purpose, MMSNs were labeled with FITC, and exposed to A549 cancer cells (adenocarcinomic human alveolar basal epithelial cells) for 1 hour. The result from confocal microscopy observation demonstrated that all cells rendered strong green fluorescence which dispersed in cytoplasm surrounding the nuclei, implying that MMSNs were successfully internalized by A549 cells (Supplementary material, Figure S2). By taking the advantage of fluorescent spectral properties of DOX (it emits fluorescence ranging from $545 \mathrm{~nm}$ to $590 \mathrm{~nm}$ when excited at about $480 \mathrm{~nm}$ wavelength), the DOX release from MMSNs to the cells can be conveniently observed. Single- or multidrug-loaded MMSNs were incubated with A549 cells for 2 hours followed by fluorescence microscopy observation (Figure 5). At this time point, the MMSNs had readily been taken up by cancer cells based on the previous results (Supplementary material, Figure S2). In fact, a time course analysis of MMSNs uptake by A549 cells indicated that internalization process occurred in as short as 15 minutes at $37^{\circ} \mathrm{C}$, while drug release occurred in 30 minutes (Supplementary material, Figure S3).

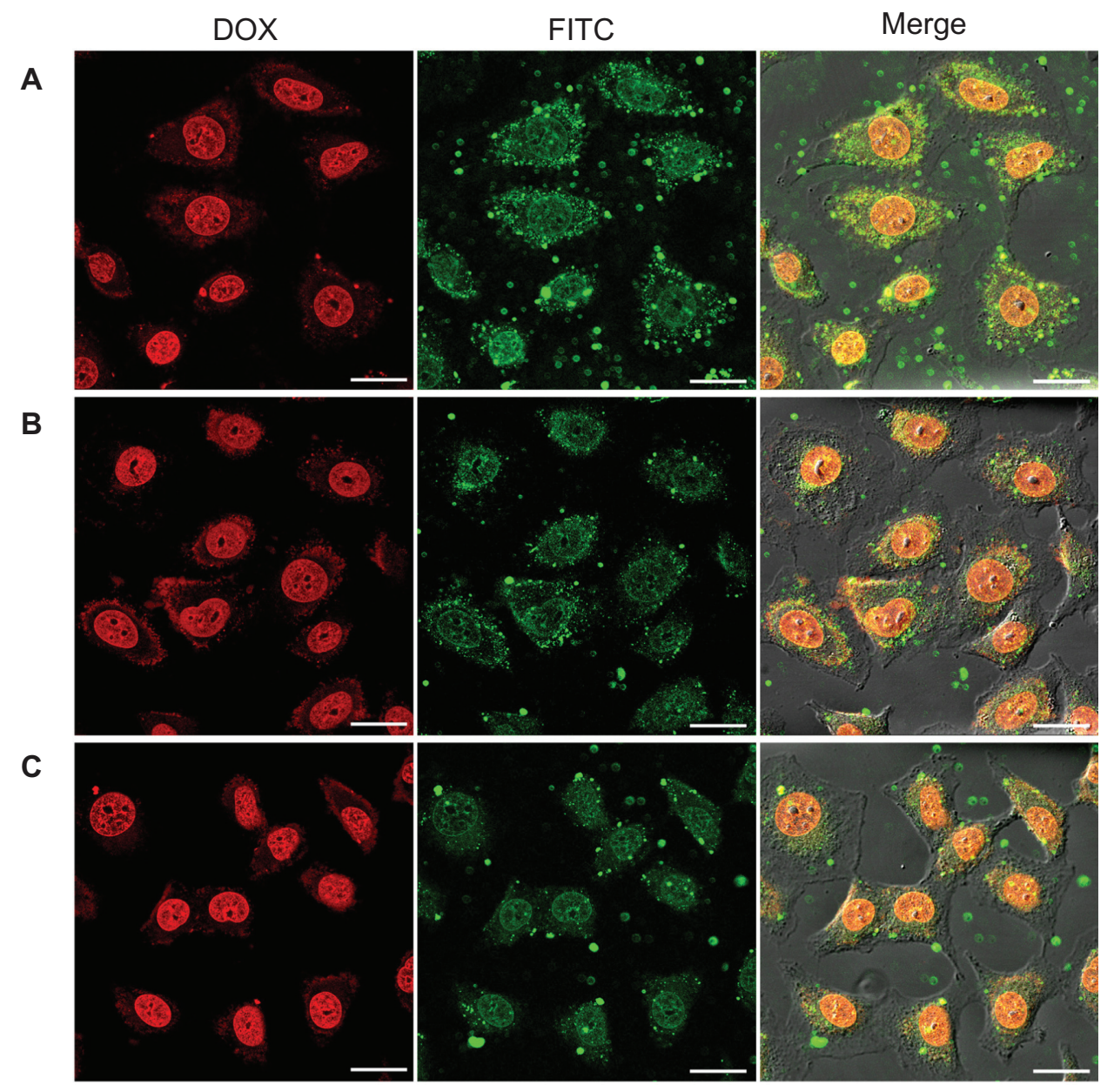

Figure 5 Intracellular distribution and drug release of drug-loaded MMSNs. A549 cells were treated by drug-loaded MMSNs for 2 hours, and then fixed and observed by confocal microscope. Both individual (A) and PTX (B) or RAPA (C) co-loaded DOX were successfully released from MMSNs, as the red fluorescence distributed in the nucleus.

Note: Scale bar: $50 \mu \mathrm{m}$.

Abbreviations: DOX, doxorubicin; MMSNs, magnetic mesoporous silica nanoparticles; PTX, paclitaxel; RAPA, rapamycin. 
The red fluorescence indicative of DOX was clearly observed inside all cells, with focused distribution in the nuclear region and scattered distribution in the cytoplasm. This is because DOX easily permeates the nuclear envelope and intercalates DNA..$^{50}$ The green fluorescence was detected in the cell plasma but also in the nuclear zone. This contradictive phenomenon of distribution of MMSNs inside the cells could be explained by the fact that DOX also emits green fluorescence when excited at $480 \mathrm{~nm}$ wavelength, which overlaps with the spectrum of FITC (Supplementary material, Figure S4). It is reported that MMSNs with size ranging from 50 to $200 \mathrm{~nm}$ were generally internalized and transported into lysosomes..$^{51}$ Exactly how the particles get internalized and transported in the cells and how the drugs get unloaded and permeate the intracellular membranous structures will require further investigation. Nonetheless, for all the three systems, drug-loaded MMSNs were successfully internalized by cancer cells and the loaded drugs could be released.

\section{Improved cancer cell killing and inhibition by the multidrug-loaded MMSNs}

Thereafter, we tested the induced effects of single- and multidrug-loaded MMSNs in the induction of cancer cell death and inhibition of growth. To keep a commensurable inhibitory effect, the loaded amount of DOX in MMSNs was controlled to be approximately $90 \mu \mathrm{g} / \mathrm{mg}$, which was chosen based on the half maximal inhibitory concentration $\left(\mathrm{IC}_{50}\right)$ of free drug in A549 cells in our pilot experiment (data not shown) and published studies. ${ }^{52}$ The ratio of DOX to PTX was scaled to 9:1 (m:m) by adopting our sequential and tunable loading strategy. Similarly, for the DOX-RAPA combination, the ratio was set to be 1:1 (m:m). We determined the percentage of cells undergoing apoptosis by flow cytometry after treatment with different drug-loaded MMSNS. Empty MMSNs without loading any drugs were included as controls. Up to $50 \mu \mathrm{g} / \mathrm{mL}$, empty MMSNs posed limited toxicity to A549 cells after 24 or 48 hours of incubation (Figures 6 and 7).

A549 cells were exposed to empty or drug-loaded MMSNs at $50 \mu \mathrm{g} / \mathrm{mL}$ for 24 hours, and stained with Annexin V-PE/7AAD, followed by flow cytometric analysis. Empty MMSNs only induced almost negligible apoptosis $(2.74 \%$ of total cells) (Figure 7), which suggests good biocompatibility. In contrast, single-drug-loaded MMSNs induced considerable apoptosis, which was 14.65\% (DOX-MMSNs), 7.64\% (PTX-MMSNs) and 8.28\% (RAPA-MMSNs) of total cells, respectively. Meanwhile, the apoptotic ratios in cells treated by multidrug-loaded MMSNs were $30.72 \%$ for DOX-PTX-MMSNs and $47.30 \%$ for DOX-RAPA-MMSNs,
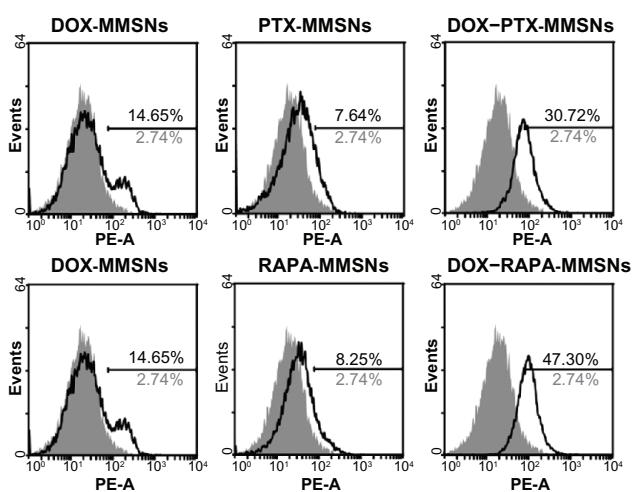

Figure 6 Apoptosis assay of A549 cells induced by single- or multidrug-loaded MMSNs. A549 cells were treated by $50 \mu \mathrm{g} / \mathrm{mL}$ empty nanoparticles (MMSNs) DOX-MMSNs, PTX-MMSNs, and DOX-PTX-MMSNs for 24 hours. The harvested cells were stained by Annexin V-PE/7AAD apoptosis kit, and the flow cytometry showed DOX-PTX-MMSNs induced more notable apoptotic effects than DOXMMSNs and PTX-MMSNs. Empty MMSNs produced a negligible effect. Grey curve represents the apoptosis of untreated cells.

Abbreviations: DOX, doxorubicin; MMSNs, magnetic mesoporous silica nanoparticles; PTX, paclitaxel; RAPA, rapamycin.

which were greater than the arithmetic sum of the apoptotic ratios induced by each single-drug-loaded MMSNs. This result implied that the combined delivery of multidrugs produced enhanced cell apoptosis.
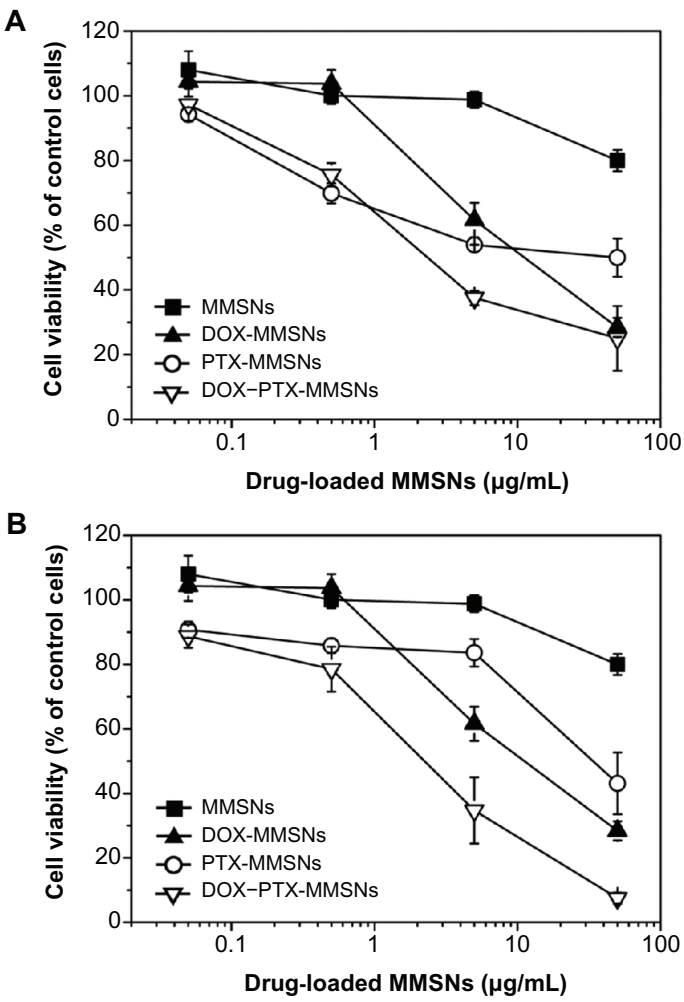

Figure 7 Tumor cell growth inhibitions induced by drug-loaded MMSNs. A549 cells were treated with empty or drug-loaded MMSNs for 48 hours. Notable cell growth suppressions were observed at: (A) $5 \mu \mathrm{g} / \mathrm{mL}$ of DOX-PTX-MMSNs and (B) $5-50 \mu \mathrm{g} / \mathrm{mL}$ of DOX-RAPA-MMSNs $(P \leq 0.05)$.

Abbreviations: DOX, doxorubicin; MMSNs, magnetic mesoporous silica nanoparticles; PTX, paclitaxel; RAPA, rapamycin. 
Furthermore, we tested the growth inhibition and toxicity effect of drug-loaded MMSNs on tumor cells in addition to the flow cytometry-based apoptosis assay. A549 cells were treated by drug-loaded MMSNs for 48 hours followed by cell viability analysis via using a CCK-8 cell counting kit (Figure 7). Empty MMSNs induced negligible toxic effect at low particle concentration, while minute toxicity was detectable at $50 \mu \mathrm{g} / \mathrm{mL}$. This might be attributed to the bare surface of the MMSNs used in our experiments, which could be eliminated by surface modification in further application. ${ }^{53}$ In contrast, both individual and combinative loading groups produced dose-dependent tumor cell growth inhibitions. Particularly, the inhibition effect of the multidrug-loaded MMSNs was more significant than that of single-drug-loaded MMSNs. Specifically, in the PTX-DOX combination (Figure $7 \mathrm{~A}$ ), the $\mathrm{IC}_{50}$ were $\sim 6.8$ and $50 \mu \mathrm{g} / \mathrm{mL}$ for DOXMMSNs and PTX-MMSNs, and $2.3 \mu \mathrm{g} / \mathrm{mL}$ for DOX-PTXMMSNs, respectively; this synergistic effect of growth inhibition was most evident at $5 \mu \mathrm{g} / \mathrm{mL}$ of MMSNs $(P \leq 0.05)$. In the RAPA-DOX combination (Figure 7B), the $\mathrm{IC}_{50}$ were $\sim 6.8$ and $28.8 \mu \mathrm{g} / \mathrm{mL}$ for DOX-MMSNs and RAPAMMSNs versus $2.5 \mu \mathrm{g} / \mathrm{mL}$ for DOX-RAPA-MMSNs; the synergistic effect of cell growth inhibition was clearly observed from 0.5 to $50 \mu \mathrm{g} / \mathrm{mL}$ of MMSNs $(P \leq 0.05)$.

Though the loading of two distinct chemotherapeutics instead of one chemotherapeutic plus the CTAB chemosensitizer as reported previously, ${ }^{33}$ our experimental data implied a more unambiguous synergistic effect in both cell apoptosis and growth inhibition. Additionally, by comparing the drug concentrations required to achieve similar levels of cell growth inhibition, we found this value for drugs loaded in MMSNs was much smaller than that for drugs in the free form (Supplementary material, Figure S5). In other words, drugloaded MMSNs produced more significant inhibition with the same amount of drugs used. Similar effects were also reported by Chang and colleagues. ${ }^{44}$ On the other hand, the DOX-PTX combination was reported to have increased antitumor activity against solid tumors, eg, lung, colon, breast, and liver cancer, ${ }^{52,54}$ while the use of the RAPA-DOX combination has yet to be documented in clinical applications. Herein we demonstrated notable synergistic anticancer effects for both DOX-PTX and DOX-RAPA combinations. Furthermore, by taking advantage of different binding sites in surfactant free mesoporous silica under the selected solvent conditions, our co-loading strategy was more general compared to He's work which was limited to drug/surfactant combinations. ${ }^{33}$ By combining the use of MMSNs for drug delivery, and choice of chemotherapeutics combinations, we have produced a feasible DDS with enhanced therapeutic efficacy in cancer treatment, which might have translatable significance.

\section{Conclusion}

In summary, we developed an approach to co-load hydrophilic and hydrophobic chemotherapeutics on MSNs, with the aim of realizing multidrug delivery for cancer therapy. Two types of chemotherapeutics combinations, ie, DOXPTX and DOX-RAPA, were successfully loaded into the $50 \mathrm{~nm}$ MSNs. The experimental data indicated that the ratio of loaded DOX versus RAPA could be easily tuned from 0:185 to $245: 64$ (m:m), while the ratio of loaded DOX versus PTX from 0:156 to 245:40 (m:m), with the overall quantity of drugs no less than $\sim 200 \mu \mathrm{g} / \mathrm{mg}$ across the full spectrum of combinations. The nanoparticles prepared by this co-loading fashion could be internalized efficiently by cancer cells, and produced an enhanced cancer cell suppression compared to individual loading. So far, this is the first endeavor that employs MSNs to load hydrophilic and hydrophobic chemotherapeutics simply by adsorbing drugs from different solvents. Our research thus realized hydrophobic-hydrophilic drugs loading, amount/ratio control, and simultaneous delivery simultaneously. Further work is needed to modify the surface of MMSNs with biocompatible polymers concerning their in vivo circulation and stabilization; and via choosing an optimal dose ratio to evaluate this co-loading fashion and the induced therapeutic effect in a tumor model. These data would facilitate multidrug combination chemotherapy.

\section{Acknowledgments}

This work was supported by National 863 High-Tech Program (2009AA03Z333), National Natural Science Foundation China (30900756), "Rising Star" Grant from Science and Technology Commission of Shanghai (09QA1403400), a start-up grant from Ministry of Education China for returnees (K10MD06), Shanghai Nano Project (1052nm01100), and Shanghai Jiao Tong University funding (YG2009ZD203, YG2010ZD102, AE4160003, YG2009MS55, YG2010MS29). The authors would like to thank the Instrumental Analysis Center of Shanghai Jiao Tong University for the characterization of materials.

\section{Disclosure}

The authors declare no conflicts of interest in this work.

\section{References}

1. Allen TM, Cullis PR. Drug delivery systems: Entering the mainstream. Science. 2004;303(5665):1818-1822. 
2. Langer R, Tirrell DA. Designing materials for biology and medicine. Nature. 2004;428(6982):487-492.

3. Brigger I, Dubernet C, Couvreur P. Nanoparticles in cancer therapy and diagnosis. Adv Drug Del Rev. 2002;54(5):631-651.

4. Brannon-Peppas L, Blanchette JO. Nanoparticle and targeted systems for cancer therapy. Adv Drug Del Rev. 2004;56(11):1649-1659.

5. Davis ME, Chen Z, Shin DM. Nanoparticle therapeutics: An emerging treatment modality for cancer. Nat Rev Drug Disc. 2008;7(9):771-782.

6. Riehemann K, Schneider SW, Luger TA, et al. Nanomedicine Challenge and perspectives. Angew Chem Int Ed. 2009;48(5):872-897.

7. Moorthi C, Manavalan R, Kathiresan K. Nanotherapeutics to overcome conventional cancer chemotherapy limitations. J Pharm Pharm Sci. 2011;14(1):67-77.

8. Vivero-Escoto JL, Slowing II, Trewyn BG, et al. Mesoporous silica nanoparticles for intracellular controlled drug delivery. Small. 2010; 6(18):1952-1967.

9. Slowing II, Vivero-Escoto JL, Wu C-W, et al. Mesoporous silica nanoparticles as controlled release drug delivery and gene transfection carriers. Adv Drug Del Rev. 2008;60(11):1278-1288.

10. Liu J, Qiao SZ, Hu QH, et al. Magnetic nanocomposites with mesoporous structures: synthesis and applications. Small. 2011;7(4):425-443.

11. Vallet-Regí M, Balas F, Arcos D. Mesoporous materials for drug delivery. Angew Chem Int Ed. 2007;46(40):7548-7558.

12. Rosenholm JM, Sahlgren C, Lindén M. Towards multifunctional, targeted drug delivery systems using mesoporous silica nanoparticles opportunities and challenges. Nanoscale. 2010;2(10):1870-1883.

13. He Q, Shi J, Zhu M, et al. The three-stage in vitro degradation behavior of mesoporous silica in simulated body fluid. Microporous Mesoporous Mater. 2010;131(1-3):314-320.

14. Lu J, Liong M, Li Z, et al. Biocompatibility, biodistribution, and drugdelivery efficiency of mesoporous silica nanoparticles for cancer therapy in animals. Small. 2010;6(16):1794-1805.

15. Luo Z, Cai K, Hu Y, et al. Mesoporous silica nanoparticles end-capped with collagen: redox-responsive nanoreservoirs for targeted drug delivery. Angew Chem Int Ed. 2011;50(3):640-643.

16. Bernardos A, Mondragon L, Aznar E, et al. Enzyme-responsive intracellular controlled release using nanometric silica mesoporous supports capped with "saccharides". Acs Nano. 2010;4(11):6353-6368.

17. Botella P, Gao F, Corma A, et al. Monodispersed mesoporous silica nanoparticles with very large pores for enhanced adsorption and release of DNA. J Phys Chem B. 2009;113(6):1796-1804.

18. Lu J, Liong M, Zink JI, et al. Mesoporous silica nanoparticles as a delivery system for hydrophobic anticancer drugs. Small. 2007;3(8): 1341-1346.

19. Lu J, Liong M, Sherman S, et al. Mesoporous silica nanoparticles for cancer therapy: Energy-dependent cellular uptake and delivery of paclitaxel to cancer cells. Nanobiotechnology. 2007;3(2):89-95.

20. Hom C, Lu J, Liong M, et al. Mesoporous silica nanoparticles facilitate delivery of siRNA to shutdown signaling pathways in mammalian cells. Small. 2010;6(11):1185-1190.

21. Li X, Xie QR, Zhang J, et al. The packaging of siRNA within the mesoporous structure of silica nanoparticles. Biomaterials. 2011;32(35): 9546-9556.

22. Slowing II, Trewyn BG, Lin VSY. Mesoporous silica nanoparticles for intracellular delivery of membrane-impermeable proteins. $\mathrm{J} \mathrm{Am} \mathrm{Chem}$ Soc. 2007;129(28):8845-8849.

23. Hanahan D, Weinberg RA. Hallmarks of cancer: the next generation. Cell. 2011;144(5):646-674.

24. Jia J, Zhu F, Ma X, et al. Mechanisms of drug combinations: interaction and network perspectives. Nat Rev Drug Discov. 2009;8(2): $111-128$

25. Gasparini G, Longo R, Fanelli M, et al. Combination of antiangiogenic therapy with other anticancer therapies: Results, challenges, and open questions. J Clin Oncol. 2005;23(6):1295-1311.

26. Fitzgerald JB, Schoeberl B, Nielsen UB, et al. Systems biology and combination therapy in the quest for clinical efficacy. Nat Chem Biol. 2006;2(9):458-466.
27. Olive KP, Jacobetz MA, Davidson CJ, et al. Inhibition of hedgehog signaling enhances delivery of chemotherapy in a mouse model of pancreatic cancer. Science. 2009;324(5933):1457-1461.

28. Hainsworth JD, Spigel DR, Burris HA, et al. Phase II trial of bevacizumab and everolimus in patients with advanced renal cell carcinoma. J Clin Oncol. 2010;28(13):2131-2136.

29. Meng H, Liong M, Xia T, et al. Engineered design of mesoporous silica nanoparticles to deliver doxorubicin and p-glycoprotein siRNA to overcome drug resistance in a cancer cell line. ACS Nano. 2010;4(8): $4539-4550$

30. Chen AM, Zhang M, Wei D, et al. Co-delivery of doxorubicin and Bcl-2 siRNA by mesoporous silica nanoparticles enhances the efficacy of chemotherapy in multidrug-resistant cancer cells. Small. 2009;5(23): 2673-2677.

31. Wang B, Rosano JM, Cheheltani Re, et al. Towards a targeted multidrug delivery approach to improve therapeutic efficacy in breast cancer. Exp Opin Drug Deliv. 2010;7(10):1159-1173.

32. Ashley CE, Carnes EC, Phillips GK, et al. The targeted delivery of multicomponent cargos to cancer cells by nanoporous particle-supported lipid bilayers. Nat Mater. 2011;10(5):389-397.

33. He Q, Gao Y, Zhang L, et al. A pH-responsive mesoporous silica nanoparticles-based multi-drug delivery system for overcoming multidrug resistance. Biomaterials. 2011;32(30):7711-7720.

34. Jingou J, Shilei H, Weiqi L, et al. Preparation, characterization of hydrophilic and hydrophobic drug in combine loaded chitosan/ cyclodextrin nanoparticles and in vitro release study. Colloids Surf B Biointerfaces. 2011;83(1):103-107.

35. Li X, Zhang J, Gu H. Adsorption and desorption behaviors of DNA with magnetic mesoporous silica nanoparticles. Langmuir. 2011;27(10): 6099-6106.

36. Zhang J, Li X, Rosenholm JM, et al. Synthesis and characterization of pore size-tunable magnetic mesoporous silica nanoparticles. $J$ Colloid Interface Sci. 2011;361(1):16-24.

37. Kim J, Kim HS, Lee N, et al. Multifunctional uniform nanoparticles composed of a magnetite nanocrystal core and a mesoporous silica shell for magnetic resonance and fluorescence imaging and for drug delivery. Angew Chem. 2008;120(44):8566-8569.

38. Brunauer S, Emmett PH, Teller E. Adsorption of gases in multimolecular layers. J Am Chem Soc. 1938;60(2):309-319.

39. Ravikovitch PI, Wei D, Chueh WT, et al. Evaluation of pore structure parameters of MCM-41 catalyst supports and catalysts by means of nitrogen and argon adsorption. $J$ Phys Chem B. 1997;101(19): 3671-3679.

40. Iyer AK, Khaled G, Fang J, et al. Exploiting the enhanced permeability and retention effect for tumor targeting. Drug Disc Today. 2006; 11(17-18):812-818.

41. Rizell M, Andersson M, Cahlin C, et al. Effects of the mTOR inhibitor sirolimus in patients with hepatocellular and cholangiocellular cancer. Int J Clin Oncol. 2008;13(1):66-70.

42. de Macedo JL, Braga PRS, Costa AA, et al. Liquid phase calorimetricadsorption analysis of Si-MCM-41: Evidence of strong hydrogen-bonding sites. Microporous Mesoporous Mater. 2011;139(1-3):74-80.

43. Luhmer M, d'Espinose JB, Hommel H, et al. High-resolution Si-29 solid-state NMR study of silicon functionality distribution on the surface of silicas. Magn Reson Imaging. 1996;14(7-8):911-913.

44. Chang B, Guo J, Liu C, et al. Surface functionalization of magnetic mesoporous silica nanoparticles for controlled drug release. J Mater Chem. 2010;20(44):9941-9947.

45. Korsmeyer RW, Gurny R, Doelker E, et al. Mechanisms of solute release from porous hydrophilic polymers. Int J Pharm. 1983;15(1):25-35.

46. Liong M, Lu J, Kovochich M, et al. Multifunctional inorganic nanoparticles for imaging, targeting, and drug delivery. ACS Nano. 2008; 2(5):889-896.

47. Seftel EM, Popovici RF, Mihai GD, et al. Controlled drug delivery system based on ordered mesoporous silica matrices of captopril as angiotensin-converting enzyme inhibitor drug. J Pharm Sci. 2011; 100(2):704-714. 
48. Andersson J, Rosenholm J, Areva S, et al. Influences of material characteristics on ibuprofen drug loading and release profiles from ordered micro- and mesoporous silica matrices. Chem Mater. 2004;16(21): 4160-4167.

49. Xu Y, Tang Q, Wu D, et al. Studies on a new carrier of trimethylsilylmodified mesoporous material for controlled drug delivery. J Controlled Release. 2006;114(1):41-46.

50. Capranico G, Kohn KW, Pommier Y. Local sequence requirements for DNA cleavage by mammalian topoisomerase II in the presence of doxorubicin. Nucleic Acids Res. 1990;18(22):6611-6619.

51. Huang DM, Hong Y, Ko BS, et al. Highly efficient cellular labeling of mesoporous nanoparticles in human mesenchymal stem cells: implication for stem cell tracking. FASEB J. 2005;19:2014-2016.
52. Jin $\mathrm{C}$, Li H, He Y, et al. Combination chemotherapy of doxorubicin and paclitaxel for hepatocellular carcinoma in vitro and in vivo. J Cancer Res Clin Oncol. 2010;136(2):267-274.

53. Di Pasqua AJ, Sharma KK, Shi YL, et al. Cytotoxicity of mesoporous silica nanomaterials. J Inorg Biochem. 2008;102(7):1416-1423.

54. Trail PA, Willner D, Bianchi AB, et al. Enhanced antitumor activity of paclitaxel in combination with the anticarcinoma immunoconjugate BR96-doxorubicin. Clin Cancer Res. 1999;5(11):3632-3638. 


\section{Supplementary information}

Table SI The loading dose ratio of DOX and PTX

\begin{tabular}{lllllll}
\hline DOX $(\mu \mathrm{g} / \mathrm{mg})$ & 0.0 & 45.9 & 93.3 & 140.5 & 186.3 & 216.2 \\
PTX $(\mu \mathrm{g} / \mathrm{mg}$, mean $\pm \mathrm{SD})$ & $156.0 \pm 3.3$ & $140.1 \pm 1.8$ & $120.4 \pm 1.0$ & $108.9 \pm 0.7$ & $79.8 \pm 1.3$ & $39.7 \pm 1.3$ \\
\hline
\end{tabular}

Abbreviations: DOX, doxorubicin; PTX, paclitaxel; SD, standard deviation.

Table S2 The loading dose ratio of DOX and RAPA

\begin{tabular}{llllll}
\hline $\operatorname{DOX}(\mu \mathrm{g} / \mathrm{mg})$ & 0.0 & 58.3 & 120.1 & 179.6 & 224.1 \\
$\operatorname{RAPA}(\mu \mathrm{g} / \mathrm{mg}$, mean $\pm \mathrm{SD})$ & $180.7 \pm 1.6$ & $124.0 \pm 2.6$ & $91.8 \pm 1.4$ & $70.9 \pm 2.1$ & $63.6 \pm 1.8$ \\
\hline
\end{tabular}

Abbreviations: DOX, doxorubicin; RAPA, rapamycin; SD, standard deviation.

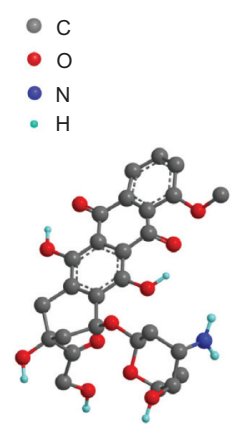

Doxorubicin

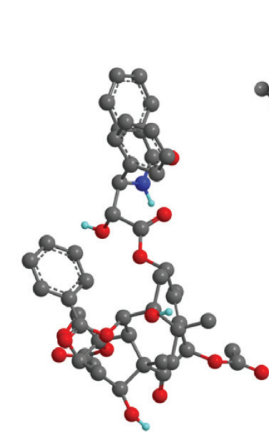

Paclitaxel

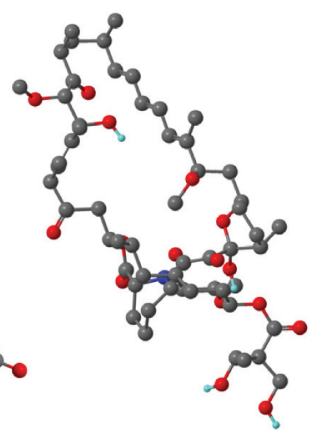

Rapamycin

Figure SI The three-dimensional molecular structures of doxorubicin, paclitaxel, and rapamycin (constructed by ChemBio 3D, molecular structure based on ChemACX database). Only polar hydrogen is shown.
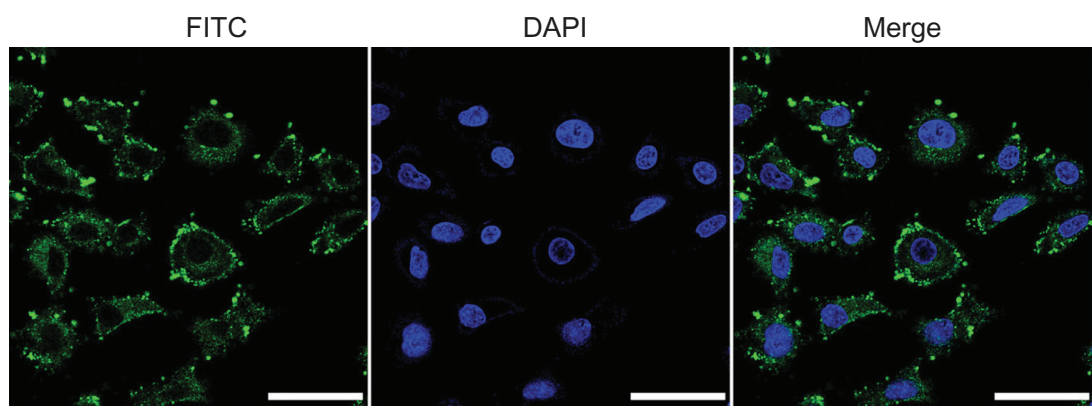

Figure S2 The cellular uptake and distribution of MMSNs on A549 cancer cells. MMSNs were labeled by FITC, and cultured with cells for I hour. Confocal microscopy image showed MMSNs were located in cytoplasm, and no green fluorescence was observed in nuclei which were stained by DAPI.

Note: Scale bar: $100 \mu \mathrm{m}$.

Abbreviations: DAPI, 4',6-diamidino-2-phenylindole; FITC, fluorescein isothiocyanate; MMSNs, magnetic mesoporous silica nanoparticles. 


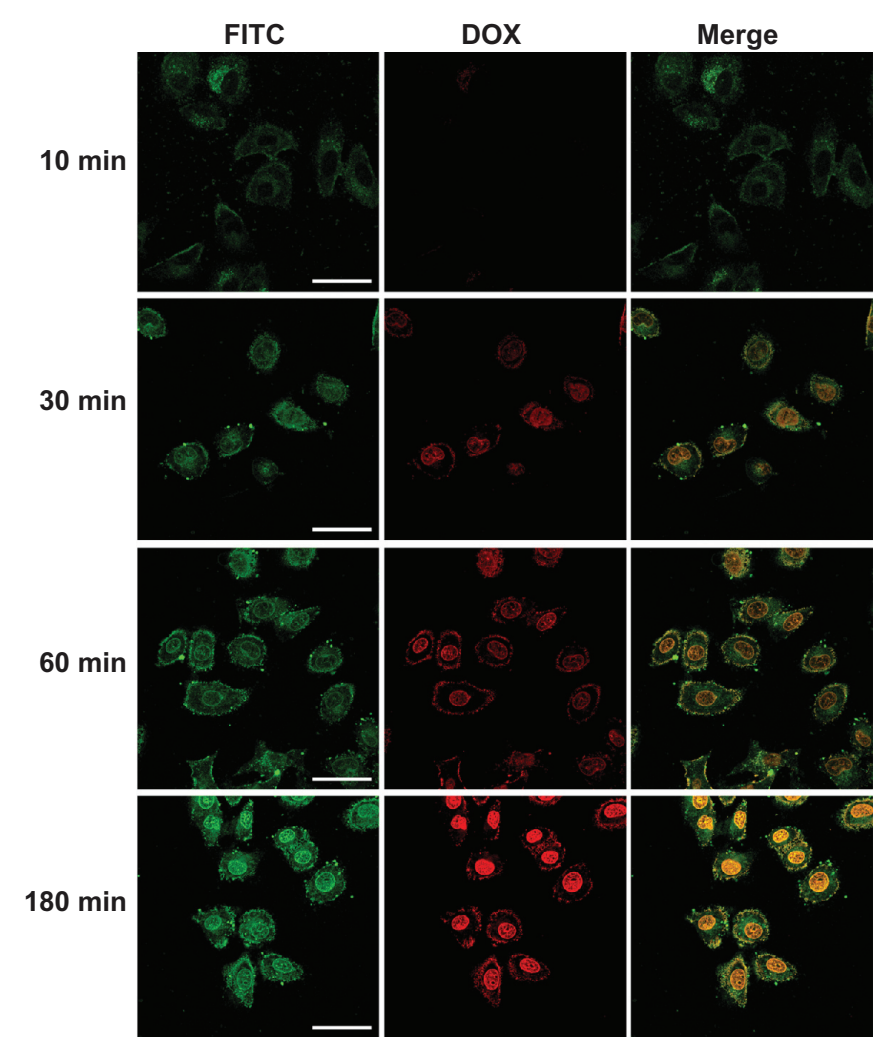

Figure S3 The cellular uptake of DOX-MMSNs and the release of DOX from MMSNs. A549 cells were treated with $50 \mu \mathrm{g} / \mathrm{mL}$ of DOX-MMSNs for I0, 30,60 , and 180 minutes, and observed by confocal microscopy. The internalization of MMSNs could be detected at the time point of 10 minutes as weak green fluorescence was observed. The fluorescence intensity increased accordingly with the prolonging of the treatment time. It was observed that the red fluorescence of DOX was almost undetectable until the incubating time increased to $30 \mathrm{~min}$, which indicated that the nanoparticles were internalized but DOX was still kept in MMSNs until the time point of 30 minutes.

Note: Scale bar: $100 \mu \mathrm{m}$.

Abbreviations: DOX, doxorubicin; FITC, fluorescein isothiocyanate; MMSNs, magnetic mesoporous silica nanoparticles.

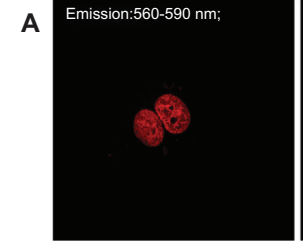

DOX

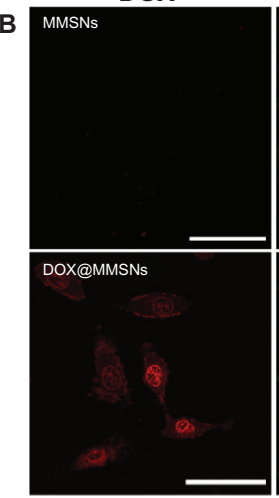

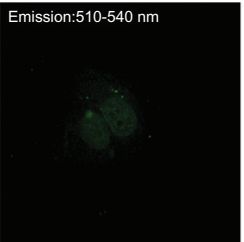

FITC

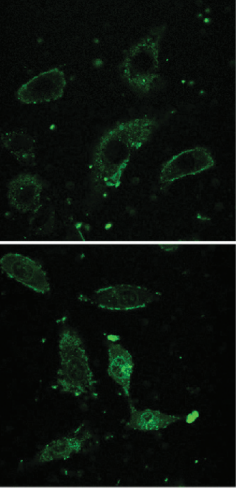

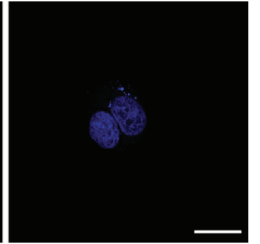

Merge

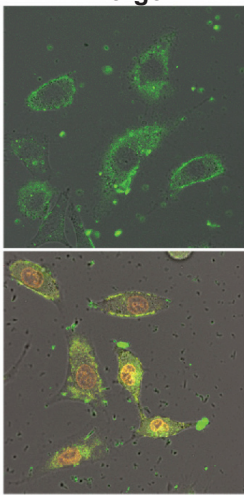

Figure S4 The fluorescence of DOX was detectable in FITC channel. (A) A549 cells were treated with free DOX in PBS at $5 \mu \mathrm{g} / \mathrm{mL}$ for I hour, fixed, and prepared for confocal microscopy. The fluorescence of DOX was mostly located in nuclei, and detectable in both 5I0-540 nm (FITC channel) and 560-590 nm emission range. (B) A549 cells were treated with empty MMSNs or DOX-MMSNs for I hour. Live cells were observed under confocal microscope. DOX-MMSNs-treated cells shows nucleic red fluorescent, whereas empty MMSNs-treated cells did not, which implies that it was the loaded DOX rather than MMSNs that entered nuclei.

Note: Scale bar: $50 \mu \mathrm{m}$.

Abbreviations: DOX, doxorubicin; FITC, fluorescein isothiocyanate; MMSNs, magnetic mesoporous silica nanoparticles. 
Free drugs $(\mu \mathrm{g} / \mathrm{mL}, 9 x$ PTX concentration)

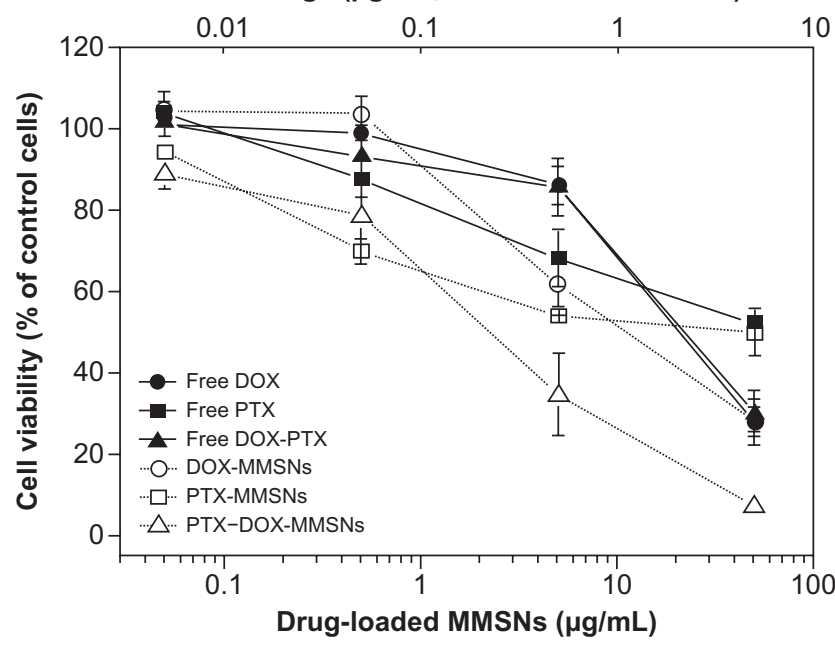

Figure S5 The growth inhibition of A549 cells induced by free or drug-loaded MMSNs with the approximate drug-loading amounts. The DOX-MMSNs loading content was $90 \mu \mathrm{g} / \mathrm{mg}$, while the PTX-MMSNs loading content was $10 \mu \mathrm{g} / \mathrm{mg}$. The cell growth inhibition induced by drugs loaded in MMSNs is more significant compared to that induced by free drugs.

Abbreviations: DOX, doxorubicin; MMSNs, magnetic mesoporous silica nanoparticles; PTX, paclitaxel.

\section{Publish your work in this journal}

The International Journal of Nanomedicine is an international, peerreviewed journal focusing on the application of nanotechnology in diagnostics, therapeutics, and drug delivery systems throughout the biomedical field. This journal is indexed on PubMed Central, MedLine CAS, SciSearch $®$, Current Contents $₫ /$ Clinical Medicine, Journal
Citation Reports/Science Edition, EMBase, Scopus and the Elsevier Bibliographic databases. The manuscript management system is completely online and includes a very quick and fair peer-review system, which is all easy to use. Visit http://www.dovepress.com/ testimonials.php to read real quotes from published authors.

Submit your manuscript here: http://www.dovepress.com/international-journal-of-nanomedicine-journal 